\title{
The visible and near infrared module of EChO
}

\author{
A. Adriani - G. Bellucci • L. Gambicorti • \\ M. Focardi • E. Oliva • M. Farina • A. M. Di Giorgio • \\ F. Santoli • E. Pace • G. Piccioni • G. Filacchione • \\ M. Pancrazzi • A. Tozzi • G. Micela
}

Received: 14 January 2014 / Accepted: 28 April 2014 / Published online: 14 June 2014

(C) The Author(s) 2014. This article is published with open access at Springerlink.com

\begin{abstract}
The Visible and Near Infrared (VNIR) is one of the modules of EChO, the Exoplanets Characterization Observatory proposed to ESA for an M-class mission. EChO is aimed to observe planets while transiting by their suns. Then the instrument had to be designed to assure a high efficiency over the whole spectral range. In fact, it has to be able to observe stars with an apparent magnitude $M_{v}=9-12$ and to see contrasts of the order of $10^{-4}-10^{-5}$ necessary to reveal the characteristics of the atmospheres of the exoplanets under investigation. VNIR is a spectrometer in a cross-dispersed configuration, covering the $0.4-2.5 \mu \mathrm{m}$ spectral range with a resolving power of about 330 and a field of view of 2 arcsec. It is functionally split into two channels respectively working in the $0.4-1.0 \mu \mathrm{m}$ and $1.0-2.5 \mu \mathrm{m}$ spectral ranges. Such a solution is imposed by the fact the light at short wavelengths has to be shared with the EChO Fine Guiding System (FGS) devoted to the pointing of the stars under observation. The spectrometer makes use of a $\mathrm{HgCdTe}$ detector of 512 by 512 pixels, $18 \mu \mathrm{m}$ pitch and working at a temperature of $45 \mathrm{~K}$ as the entire VNIR optical bench. The instrument has been interfaced to the telescope optics by two optical fibers, one per
\end{abstract}

A. Adriani · G. Bellucci $(\bowtie) \cdot$ M. Farina · A. M. Di Giorgio · F. Santoli • G. Piccioni • G. Filacchione INAF Istituto di Astrofisica e Planetologia Spaziali, Via del Fosso del Cavaliere, 00133 Rome, Italy e-mail: giancarlo.bellucci@iaps.inaf.it

L. Gambicorti $\cdot$ M. Focardi $\cdot$ E. Oliva $\cdot$ M. Pancrazzi $\cdot$ A. Tozzi

INAF Osservatorio Astrofisico di Arcetri, Largo E. Fermi 5, 50125 Florence, Italy

E. Pace

Dipartimento di Fisica e Astronomia, Università degli Studi di Firenze, Largo E. Fermi 2, 50125 Florence, Italy

M. Farina $\cdot$ G. Micela

INAF Osservatorio Astronomico di Palermo, Piazza del Parlamento 1, 90134 Palermo, Italy

Present Address:

L. Gambicorti

Physics Institute Space Research and Planetary Sciences, Sidlerstrasse 5, 3012 Bern, Switzerland 
channel, to assure an easier coupling and an easier colocation of the instrument inside the EChO optical bench.

Keywords Exoplanets · Transit spectroscopy $\cdot$ Astrobiology $\cdot$ Space astronomy

\section{Introduction}

The discovery of over a thousand exoplanets has revealed an unexpectedly diverse planet population. We see gas giants in few-day orbits, whole multi-planet systems within the orbit of Mercury, and new populations of planets with masses between that of the Earth and Neptune-all unknown in the Solar System. Observations to date have shown that our Solar System is certainly not representative of the general population of planets in our Milky Way [1]. The key science questions that urgently need addressing by EChO are therefore: What are exoplanets made of? Why are planets as they are? How do planetary systems work and what causes the exceptional diversity observed as compared to the Solar System? The EChO mission [2] will take up the challenge to explain this diversity in terms of formation, evolution, internal structure and planet and atmospheric composition. This requires in-depth spectroscopic knowledge of the atmospheres of a large and well-defined planet sample for which precise physical, chemical and dynamical information can be obtained.

In order to fulfill this ambitious scientific programme, EChO is designed as a dedicated survey mission for transit and eclipse spectroscopy capable of observing a large, diverse and well-defined planet sample within its 4-year mission lifetime. The transit and eclipse spectroscopy method, whereby the signal from the star and planet are differentiated using knowledge of the planetary ephemerides, allows us to measure atmospheric signals from the planet at flux levels of at least $10^{-4}$ relative to the star. This can only be achieved in conjunction with a carefully designed stable payload and satellite platform. It is also necessary to provide an instantaneous broad-wavelength coverage to detect as many molecular species as possible, to probe the thermal structure of the planetary atmospheres and to correct for the contaminating effects of the stellar photosphere. This requires wavelength coverage of at least 0.55 to $11 \mu \mathrm{m}$ with a goal of covering from 0.4 to $16 \mu \mathrm{m}$. Only modest spectral resolving power is needed, with $R \sim$ 300 for wavelengths less than $5 \mu \mathrm{m}$ and $R \sim 30$ for wavelengths greater than this. The transit spectroscopy technique means that no spatial resolution is required. A telescope collecting area of about $1 \mathrm{~m}^{2}$ is sufficiently large to achieve the necessary spectrophotometric precision: in practice the telescope will be $1.13 \mathrm{~m}^{2}$, diffraction limited at 3 $\mu \mathrm{m}$. Placing the satellite at L2 provides a cold and stable thermal environment as well as a large field of regard to allow efficient time-critical observation of targets randomly distributed over the sky. EChO is designed, without compromise, to achieve a single goal: exoplanet spectroscopy. The spectral coverage and signal-to-noise ratio to be achieved by EChO, thanks to its high stability and dedicated design, will be a game changer by allowing atmospheric compositions to be measured with unparalleled exactness: at least a factor 10 more precise and a factor 10 to 1,000 more accurate than current observations. This will enable the detection of molecular abundances three orders of magnitude lower than currently possible. Combining these data with estimates of planetary bulk compositions from accurate measurements of their radii and masses 
will allow degeneracies associated with planetary interior modeling to be broken, giving unique insight into the interior structure and elemental abundances of these alien worlds.

EChO will carry a single, high stability, spectrometer instrument. The baseline instrument for EChO is a modular, three-channel, highly integrated, common field of view, spectrometer that covers the full EChO required wavelength range of $0.55 \mu \mathrm{m}$ to $11.0 \mu \mathrm{m}$. The baseline design includes the goal wavelength extension to $0.4 \mu \mathrm{m}$ while an optional LWIR channel extends the range to the goal wavelength of $16.0 \mu \mathrm{m}$. Also included in the payload instrument is the Fine Guidance System (FGS), necessary to provide closed-loop feedback to the high stability spacecraft pointing. The required spectral resolving powers of 300 or 30 are achieved or exceeded throughout the band. The baseline design largely uses technologies with a high degree of technical maturity.

The spectrometer channels share a common field of view, with the spectral division achieved using a dichroic chain operating in long-pass mode. The core science channels are a cross-dispersed spectrometer VNIR module covering from 0.4 to $\sim 2.5 \mu \mathrm{m}$, a grism spectrometer SWIR module covering from 2.5 to $5.3 \mu \mathrm{m}$, and a prism spectrometer MWIR module covering from 5.3 to $11 \mu \mathrm{m}$. All science modules and the FGS are accommodated on a common Instrument Optical Bench. The payload instrumentation operates passively cooled at $\sim 45 \mathrm{~K}$ with a dedicated instrument radiator for cooling the FGS, VNIR and SWIR detectors to $40 \mathrm{~K}$. An Active Cooler System based on a Neon Joule-Thomson Cooler provides the additional cooling to $\sim 28 \mathrm{~K}$ which is required for the longer wavelength channels.

In the following, the characteristics of the VNIR module are described in detail.

\section{Scientific and technical requirements}

The VNIR design must fulfill both scientific and technical requirements imposed by the Echo mission. Spectroscopy of planetary transits of a large variety of exoplanets required the use of a multichannel spectrometers to cover the wide wavelength range. The EChO payload is therefore constituted by 3 modules (VNIR, SWIR and MWIR); moreover, a quite low operational temperature is needed to operate both SWIR and MWIR detectors and to reduce the background noise. Photometric stability and SNR are also crucial parameters in order to assure the scientific objectives of the mission. Table 1 summarize the most important VNIR requirements. The complete list can be found in EChO Mission Requirements Document [3].

\section{Module design}

\subsection{Optical layout}

The system covers the spectral range between 0.4 and $2.5 \mu \mathrm{m}$ without gaps and the resulting resolving power is nearly constant, $R \approx 330$. The wide spectral range is achieved through the combined use of a grating with a ruling of 14.3 grooves $/ \mathrm{mm}$ and blaze angle of $3.3^{\circ}$ for wavelength dispersion in horizontal direction and an order sorting calcium fluoride prism (angle $22^{\circ}$ ), which separates the orders along the vertical 
Table 1 Main EChO VNIR module scientific and technical requirements

$\Delta \mathrm{N}$ is the noise increase due to pointing inaccuracy and postprocessing. $\mathrm{N}$ is the fundamental white noise floor (i.e. photon noise from the target and zodiacal background)

\begin{tabular}{ll}
\hline Parameter & Value \\
\hline Spectral range & $0.55-2.47 \mu \mathrm{m}$ (goal 0.4-2.5 $\mu \mathrm{m})$ \\
Resolving power & $\geq 300$ \\
SNR & $\geq 200$ for a star Mv $=5$ \\
Photometric stability & $\Delta \mathrm{N} \leq 10 \% \mathrm{~N}$ in the $3.8 \times 10^{-} 6 \mathrm{~Hz}$ to \\
& $16 \mathrm{mHz}$ frequency band \\
Temporal resolution & $\leq 30 \mathrm{~s}$ \\
Absolute calibration & $5 \%$ \\
Working temperature & $40-45 \mathrm{~K}$ \\
\hline
\end{tabular}

direction. The collimator (M1) and the prism are used in double pass (see Fig. 1). The prism is the only optical element used in transmission. All other optics are made of reflecting surfaces: 2 off-axis conic mirrors, 1 spherical mirror, 1 flat mirror and 1 grating. All reflecting elements will be made of the same aluminium alloy as the optical bench. This simplifies the mechanical mount and alignment of the system. The light is fed to the spectrometer via two fibres positioned on the side of the M2 mirror. The fibres are commercial, radiation resistant, space qualified, fused-silica with ultra-low $\mathrm{OH}$ content and core diameter of $50 \mu \mathrm{m}$. Their internal absorption is lower than $1 \mathrm{db} / \mathrm{m}$ up to $2.4 \mu \mathrm{m}$, and reaches $2 \mathrm{db} / \mathrm{m}$ at $2.5 \mu \mathrm{m}$. Therefore, by limiting their length to 0.2 $\mathrm{m}$, one can achieve an internal transmission $>90 \%$ over the full wavelength range. The

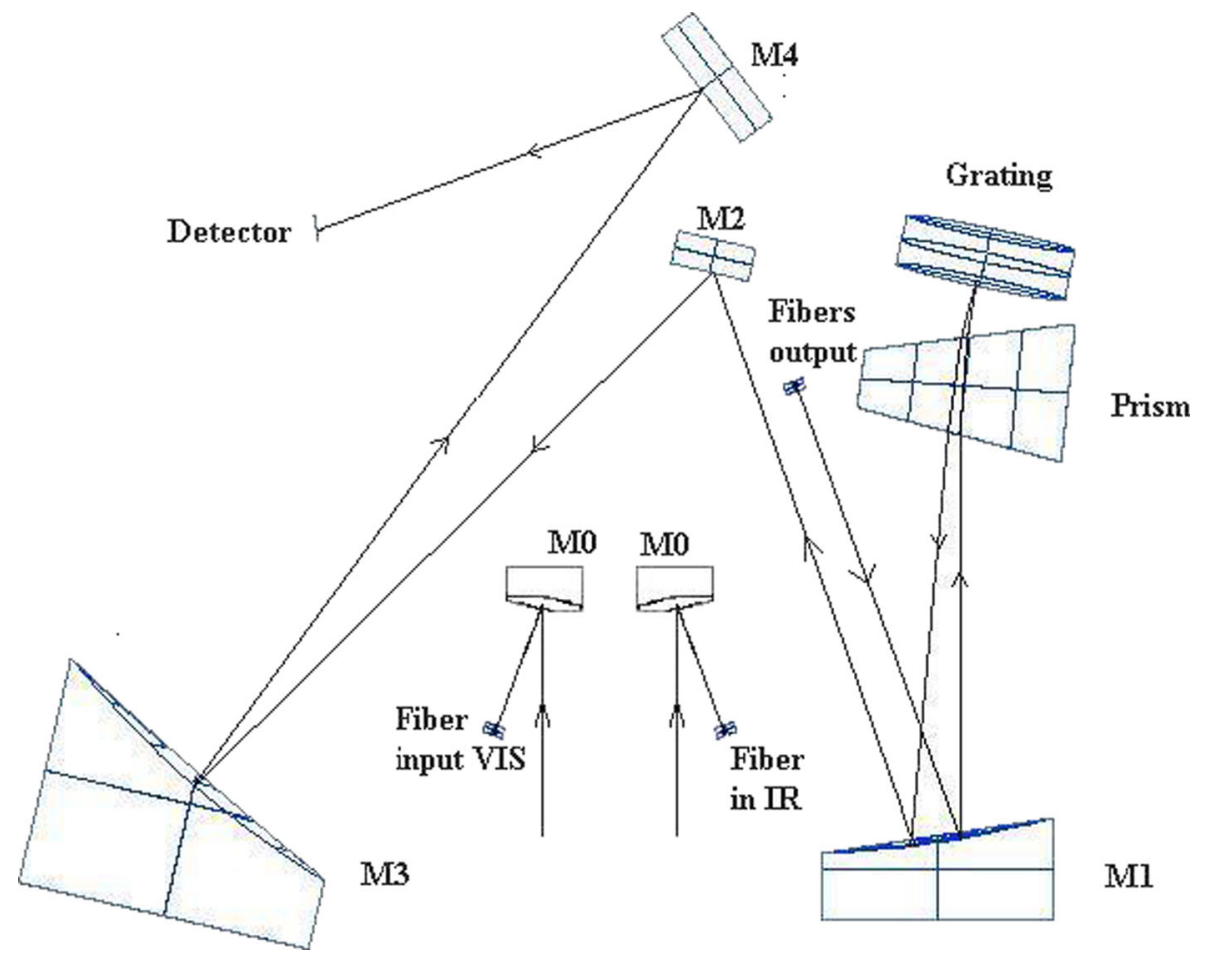

Fig. 1 Optical layout of the VNIR spectrometer 
fibres are separately fed by two identical off-axis parabolic mirrors (M0) which intercept the collimated light transmitted from the first dichroic (D1b), IR, and reflected by the beam-splitter, VIS. The use of an optical fibres coupling gives a larger flexibility in the location of the VNIR spectrometer within the EChO payload module. The VNIR characteristics are summarized in Table 2.

A Mercury Cadmium Telluride (MCT, HgCdTe) detector has been considered for VNIR (its technical characteristics are detailed later in section 4). Figure 2 shows the observable spectral orders, $\mathrm{m}$, projected on the MCT array, starting from $m=3$ at the bottom (near infrared spectral range) to $m=20$ on the top (visual spectral range).

Namely, the figure shows the distribution of the light on the array between 2,500 nm $(m=3)$ and $400 \mathrm{~nm}(m=20)$. The central wavelength in each order $m$, positioned at the blaze angle of the grating, is given by the relationship $\lambda=8.1 / m \mu \mathrm{m}$. The VIS and IR spectral ranges are separated on the detector because the fibres are placed at the spectrometer entrance are separated by $1 \mathrm{~mm}$. In general, most wavelengths are sampled twice on different orders, i.e. in different areas of the detector, as shown in Fig. 2. The spectrum in each order is spread across several pixels in the vertical

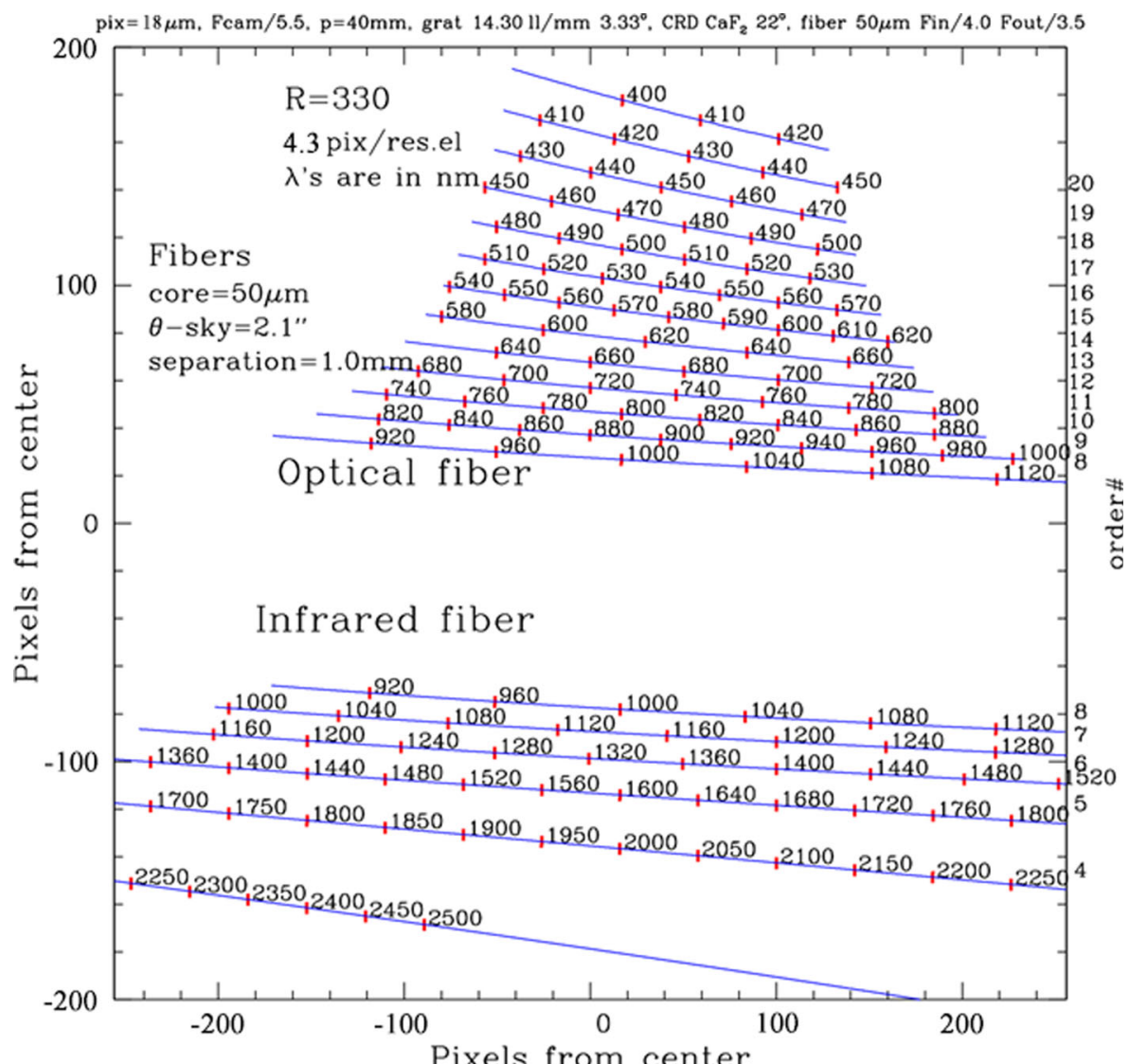

Fig. 2 Grating diffraction orders projected on the VNIR detector, starting from $m=3$ at the bottom (near infrared spectral range) to $m=20$ on the top (visual spectral range). The wavelengths in nanometers are also indicated 
direction. Thus, a sum over 5 pixels will be done to increase the sensitivity of the system in order to provide a so-called spectral channel. The last two instrumental features, about wavelength sampling, also have the advantage to reduce systematic errors in the measurements once properly exploited.

As previously said, the coupling of the VNIR module to the telescope will be done through the use of a dichroic element that will select and direct the visible and near infrared light towards the combined system VNIR and FGS. A beam-splitter is foreseen to further divide the light beam between FGS and VNIR. The balance of this beamsplitter will need to be studied in conjunction with the FGS team during the assessment phase to maximize the science return while maintaining sufficient signal for the guider system. As the performance of the module optics should be very good to assure the observations of transient planets in transit or in occultation of a star, the detector is going to be a key element in the system. In order to meet the EChO visible channel performance requirements, it is possible to pursue different ways, based on different detectors and readout electronics as well as on the optical spectrometer design characteristics.

\subsubsection{Internal calibration unit}

The instrument calibration is going to be performed looking at a known reference star before and after any target observation. The star calibration is meant to verify mainly the position of the spectral lines but also the radiometric response. A very high level of radiometric accuracy, better than $10^{-4}$, is assured by the continuous monitoring of the mother star during the transit observations. The observation session is supposed to vary from minutes to about $10 \mathrm{~h}$ depending on the characteristic of the target itself. However, as standalone procedure regardless any request for a star pointing, it is important to monitor also the stability of the instrument and, in particular, of the detector along the mission. For this purpose, a less demanding accuracy and stability is needed, of the order of a few percent. The calibration unit will be equipped with two HalogenTungsten lamps for redundancy. These kinds of lamps are currently used as spectral calibration sources of optical systems (see $[4,5]$ ) and they are the baseline for the development of the VNIR calibration unit too. The calibration lamps will be equipped

Table 2 Main EChO VNIR module characteristics

\begin{tabular}{ll}
\hline Parameter & Value \\
\hline Spectral range & $0.4-2.5 \mu \mathrm{m}$ \\
Resolving power & $\approx 330$ \\
FOV & $2 \mathrm{arcsec}$ \\
SNR & $25 @ 1.5 \mu \mathrm{m}$ for $1 \mathrm{~s}$ exposure, \\
& star Mv=9 \\
Detector type & $\mathrm{HgCdTe}$ \\
Detector size & $512 \times 512$ pixels \\
Pixel size & $18 \mu \mathrm{m} \mathrm{square}$ \\
Pixel binning & $5 \times 5$ \\
Signal digitalization & $16 \mathrm{bits}$ \\
Working temperature & $40-45 \mathrm{~K}$
\end{tabular}


with a close loop control system to assure the requested stability over the observation time. The lamps will have color temperature higher that $3,000 \mathrm{~K}$ and they will be operating for very short times during the observation sessions. The signal coming from one of the two lamps, can be used to perform several instrumental checks during the development of the mission: to verify the in-flight stability of the instrumental spectral response and registration; to perform a check on the relative radiometric response of the instrument; to monitor the evolution of possible defective pixels. The lamps inject their light into an integration sphere, which will have two output fibers that will feed the two input fibers to the spectrometer (ranges $0.4-1.0 \mu \mathrm{m}$ and $1.0-2.5 \mu \mathrm{m}$ respectively). Figure 3 gives the spectrum in input to the fibers. The feeding of the main fibers will be done using 2 in-1 out fiber connectors. The two fibers will be illuminated at the same time.

The calibration unit will be located in a separate box on a side of the service box where the mirrors collect the light from the VNIR feeding optics and focus it on the optical fibers inputs. Figure 4 shows the calibration unit and its arrangement on the service side of the VNIR optical bench.

\subsection{Mechanical and thermal design}

VNIR instrument is housed in a mechanical structure, that will be flat-mounted on the spacecraft interface (an alternative isostatic mounting could be evaluated if needed to reduce optical bench distortions). The optical elements (mirrors and prism) are shown in the right panel of Fig. 5. The figure shows the box without the calibration unit mounted below the spectrometer optical bench. A view inside the box is given in the right panels of the figure where the location of the optical elements of the spectrometer is shown. The lower part of the VNIR optical bench will be dedicated to the services to spectrometer: the input box where the mirrors concentrate the light on the optical fibers and the calibration unit in two separated box in order to minimize light and thermal contamination of the rest of the instrument. The VNIR calibration unit switches on/off and overall control will be performed by the EChO Instrument Control Unit (ICU) [6]. The mass of the instrument is estimated to be about $6.62 \mathrm{~kg}$ ( $20 \%$ margin included). The overall dimensions are: $342 \times 325 \times 190 \mathrm{~mm}$ as depicted in the lower left panel of Fig. 5. The VNIR First Resonant Frequency is planned to be larger than $150 \mathrm{~Hz}$.

Fig. 3 Spectrum of the internal calibration lamp used to check the instrument stability

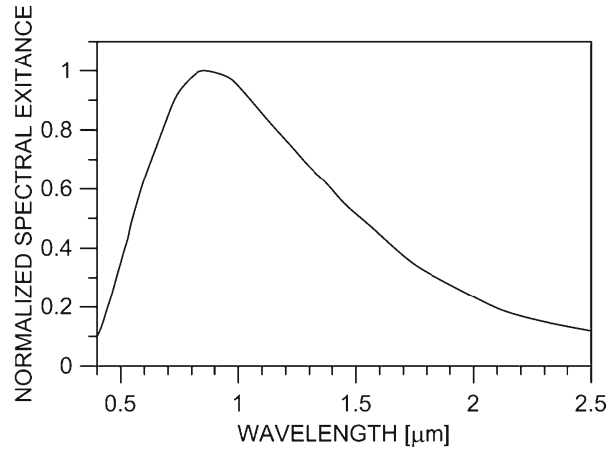




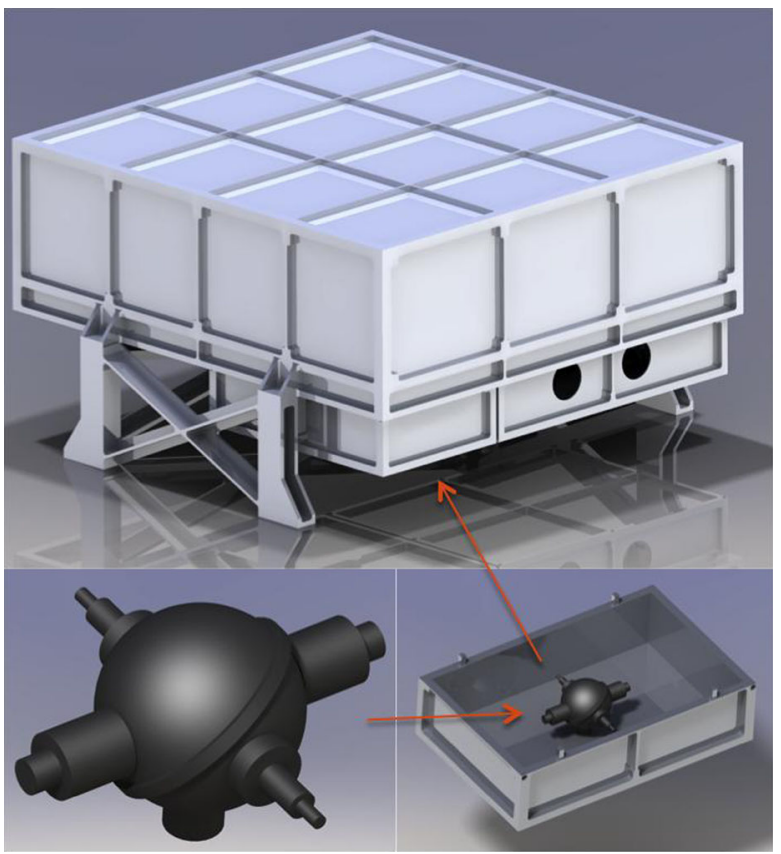

Fig. 4 Sketch of the internal calibration unit. The calibration unit is equipped with two halogen-tungsten lamps for redundancy. The lamps inject their light into an integration sphere, having two output fibers that will feed the two input fibers to the spectrometer (ranges $0.4-1.0 \mu \mathrm{m}$ and $1.0-2.5 \mu \mathrm{m}$ respectively). The calibration unit is located in a separate box on a side of the service box where the mirrors collect the light from the VNIR feeding optics and focus it on the optical fibers inputs

VNIR CFEE (Cold Front End Electronics-SIDECAR ASIC as baseline) will be located on the telescope optical bench; these are supposed to be at temperatures lower than $50 \mathrm{~K}$; the detector is planned to work at a temperature in a range of 40-45 $\mathrm{K}$, dissipating about $30 \mathrm{~mW}$. In order to minimize the thermo elastic deformations and assure a good performance also at low temperatures, the instrument (optical bench, optical supports and mirrors substrates) will be realized in the same material of the payload optical bench (aluminium) and the box will be thermally linked through its feet to it.

\section{Instrument performances}

The grating's orders of diffraction, as shown in Fig. 2, on the detector would not be equally illuminated if the input light would have a constant intensity over the entire spectrum because the grating's efficiency changes along the order. The maximum efficiency is around the center of the blue curves in Fig. 2. In this spectrometer configuration some wavelengths can be observed on two adjacent diffraction orders. To completely recover the light at those wavelengths the signal coming from the adjacent order has to be summed. The sum has to be done to maximize the result and keep the highest feasible signal to noise ratio. A reasonable compromise has been found in summing the adjacent orders when the grating efficiency is higher than $80 \%$ 

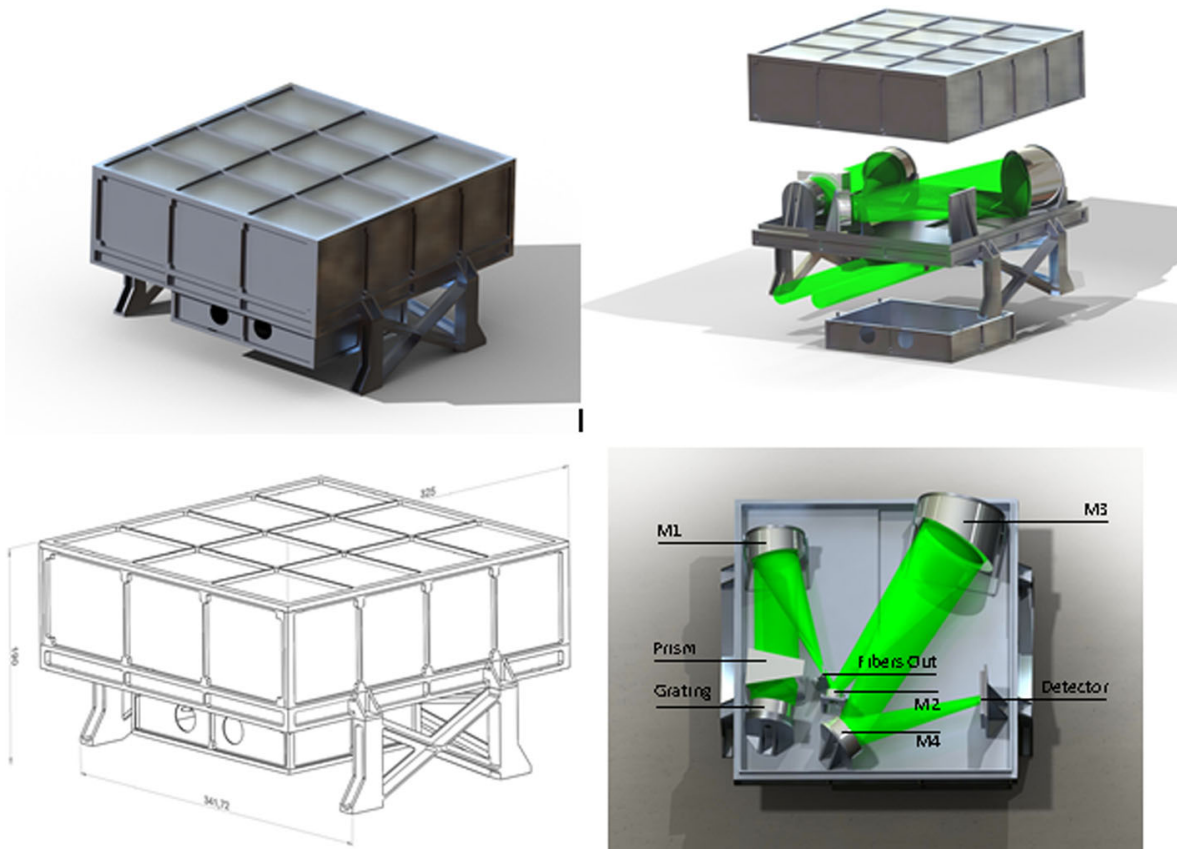

Fig. 5 VNIR box close (left panels), open (upper right panel) without the internal calibration unit and top view of the spectrometer elements (lower right panel). The path of the light inside the instrument is shown in green

with respect to the maximum. The result is a component of the Instrument Transfer Function (ITF) that will be given as result of the on-ground instrumental calibrations by measuring and combining the optical efficiency of the spectrometer and the detector performances. Figure 6 shows the spectrometer efficiency calculated with $80 \%$ criterion. The present calculation has been done by considering aluminum mirrors without any coating to improve the performances at wavelengths lower than $1.0 \mu \mathrm{m}$. In the picture the expected behavior obtained by the use of coated aluminum or protected silver mirrors is also shown for comparison.

The photometric stability is a key factor in the noise budget of the observations. The photometric stability of the instrument throughout consecutive observations lasting up to tens of hours (to cover the goal of phase curve observations) is mainly governed by the following factors:

a) Pointing stability of the telescope quantified in terms of Mean Performance Error (MPE), Pointing Drift Error (PDE) and Relative Performance Error (RPE), see below for details;

b) Thermal stability of the optical-bench and mirrors: thermal emission of the instrument can be regarded as negligible for most wavelengths, but become observable at wavelengths beyond $12 \mu \mathrm{m}$. The stability payload module (instrument and telescope) is therefore an important factor for the photometric stability in MWIR and LWIR channels.

c) Stellar noise and other temporal noise sources: whilst beyond the control of the instrument design, noise is an important source of temporal instability in 


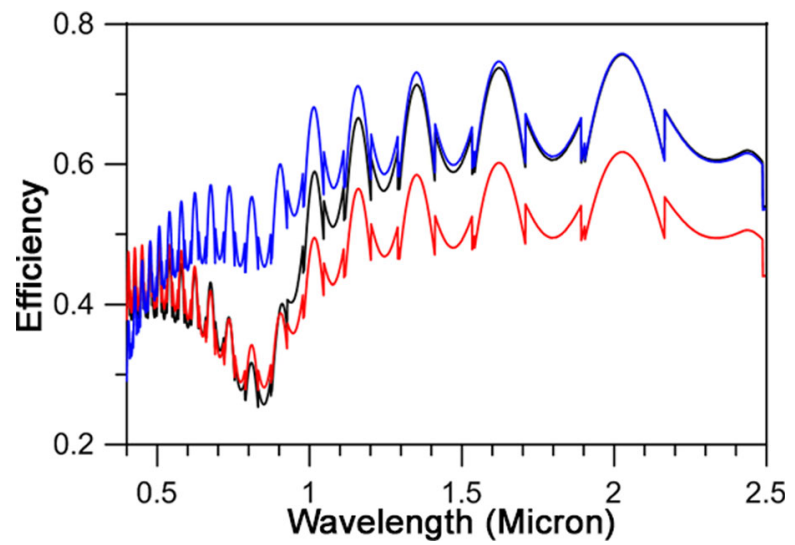

Fig. 6 VNIR efficiency: black curve, present estimation (this study); red curve: estimation done by using coated aluminium for improving the relative efficiency below $1 \mu \mathrm{m}$; blue curve, estimation done by using protected silver for all the mirrors

exoplanetary time series measurements. This is particularly true for $\mathrm{M}$ dwarf host stars as well as many non-main sequence stars. Correction mechanisms of said fluctuations must and will be an integral part of the data analysis of EChO [7].

As mentioned above, the pointing stability is affected by the following jitter types: Relative Performance Error (RPE), defining the high frequency $(>1 \mathrm{~Hz})$, unresolved jitter component. Performance Reproducibility Error (PRE), defining the low frequency $(<1 \mathrm{~Hz})$, resolved PSF drifting due to pointing jitter and Mean Performance Error (MPE) which is the overall offset (in time series, the flux offset) between two or more observation windows. The effect of the relative performance error (RPE) is a photometric error within an observation while the effect of the mean performance error (MPE) is a loss of efficiency from observation to observation. To quantify the effects of jitter on the observations, a simulation has been performed at two representative wavelengths $(0.8$ and $2.5 \mu \mathrm{m})$. The illumination pattern of the telescope is obtained from optical modeling. The energy collected by the fiber is then studied as a function of MPE, RPE and PRE. The MPE is varied in accordance to EIDA-R-0470. The impact of three different RPEs is studied: i) RPE1 $=30$ mas-rms from 1 to $10 \mathrm{~Hz}$; ii) RPE2 $=50$ mas-rms from 1 to $300 \mathrm{~Hz}$; iii) RPE3 = 130 mas-rms from 1 to $300 \mathrm{~Hz}$. These three cases correspond to three different AOCS (Attitude and Orbit Control Systems) solutions. A fixed PRE $=20$ mas-rms from 0.020 to $4 \mathrm{mHz}$ is used in this simulation.

The results of the simulations are discussed in [8] and are here briefly summarized. The effect of the MPE on the normalized transmitted energy is shown in Fig. 7. The combined effect of the RPE and PRE on the photometric error is shown in Fig. 8. The worst case photometric error is obtained when observing a bright target (a star with visual magnitude $\mathrm{Mv}=4$ ) with the RPE3 option and results in $10 \%$ of the total allowed system noise variance in $1 \mathrm{~s}$ of integration for this channel.

The analysed optical system is the Echo Telescope and the concentrating system (f\#4) in input of fibre. The configuration optimized consists in primary mirror telescope distance $\mathrm{M}_{\mathrm{T} 1}-\mathrm{M}_{\mathrm{T} 2}=1.500 \mathrm{~mm}$, the configuration defocused determines WFE $250 \mathrm{rms}$ with shift M1-M2 position of $87 \mu \mathrm{m}$. (WFE calculated at $1 \mu \mathrm{m}$ wavelength). The fibre with $50-\mu \mathrm{m}$ diameter corresponds to a Field of View (FOV) of 2 arcsec. 


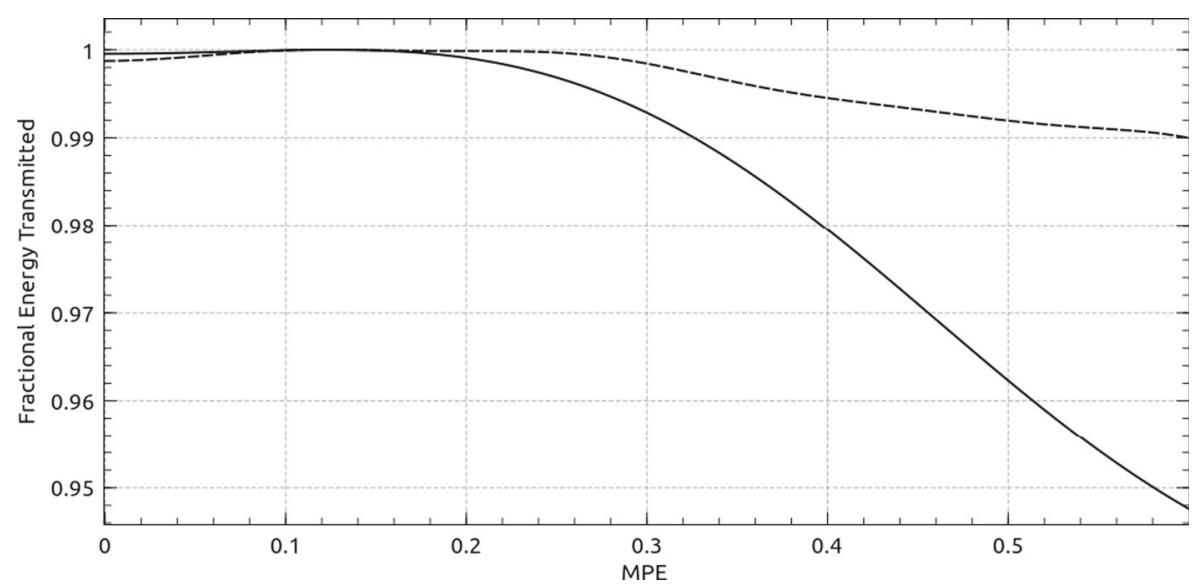

Fig. 7 Normalized energy loss vs MPE at $0.8 \mu \mathrm{m}$ (dashed line) and $2.5 \mu \mathrm{m}$ (solid line)

The spot diagram and the encircled energy are simulated to verify the requirement. Simulation of encircled energy in $250 \mathrm{rms}$ WFE generated by defocusing $\left(\mathrm{M}_{\mathrm{T} 1}\right.$ back with respect to $\left.\mathrm{M}_{\mathrm{T} 2}\right)$ of $0.087 \mathrm{~mm}\left(\mathrm{FOV} / 2=1 \operatorname{arcsec}\left(2.7 \times 10^{-5} \mathrm{deg}\right)\right.$. (The simulation considers only defocusing shift on optical axis. It is not a complete evaluation of efficiency because the tilt and lateral shift are not included).

Figure 9 shows that the spot diagram of the aberrated beam after defocusing is collected inside the fiber diameter.

Table 3 resumes all obtained results, spot diagram and Encircled Energy collected on entrance fiber of VNIR channel.

Spot diagram inside the fibre diameter and collected Encircled Energy (96.75\%) demonstrate that the introduction of a defocusing of 250 WFE rms in entrance beam of fibre.

The efficiency of a fiber is the product of three effects, namely internal transmission (which is at most $95 \%$ in our case), reflection losses at the entrance/exit (which amount
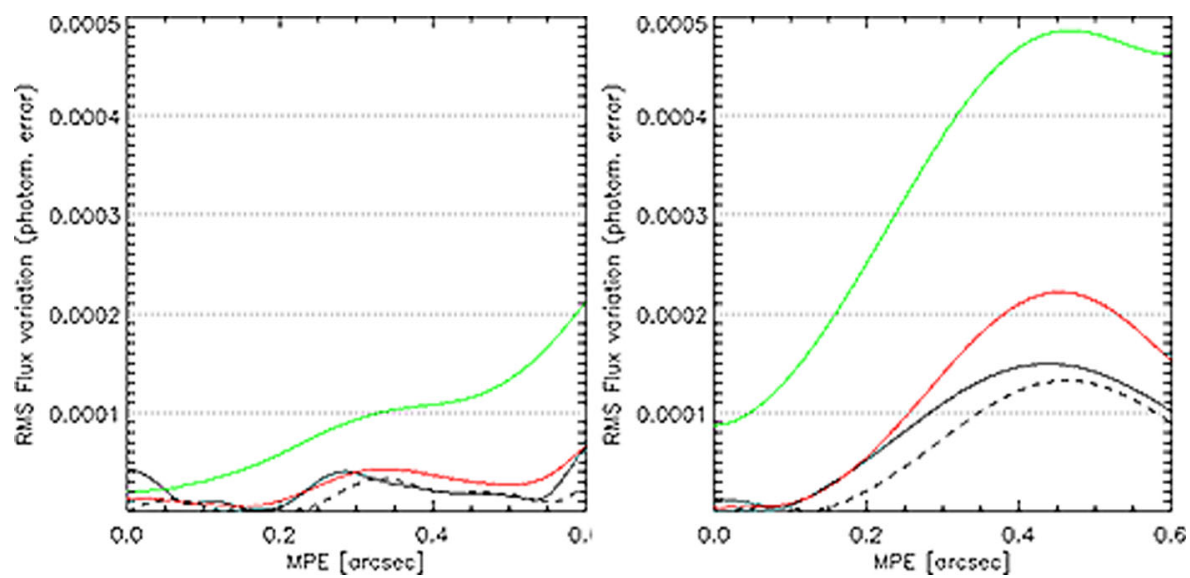

Fig. 8 Photometric error induce by the combine RPE/PRE at $0.8 \mu \mathrm{m}($ left) and $2.5 \mu \mathrm{m}$ (right) in $1 \mathrm{~s}$ of integration. The solid lines correspond to the RPE1 (black), RPE2 (red) and RPE3 (green) cases discussed in the text 


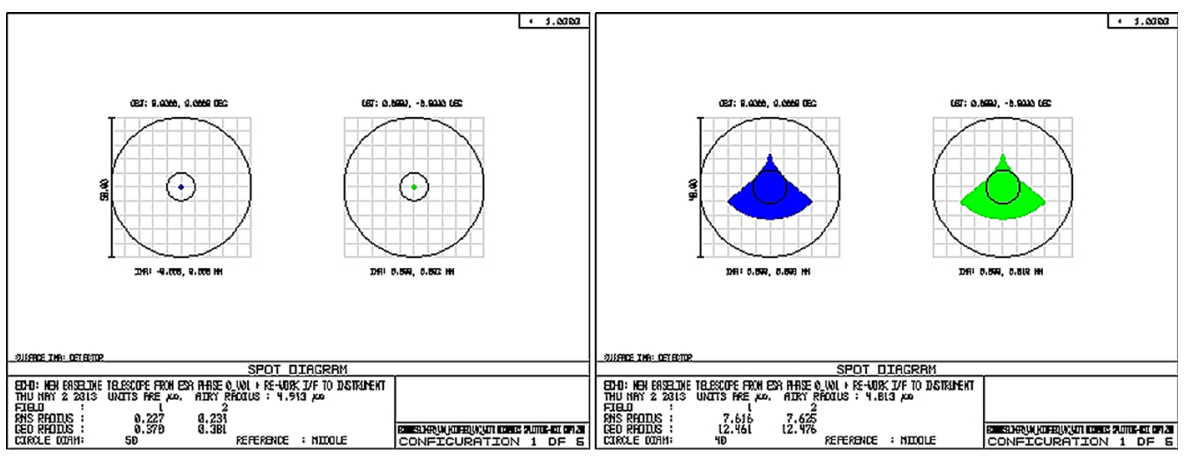

Fig. 9 Spot diagram of focused system in $50 \mu \mathrm{m}$ at central field $0.0^{\circ}$ in blue and marginal field $0.00000278^{\circ}$ in green colour (on left side) and Spot diagram of defocused system in $50 \mu \mathrm{m}$ at central field $0.0^{\circ}$ in blue and marginal field $0.00000278^{\circ}$ in green colour (right side)

to $6 \%$ ) and focal ratio degradation (FRD), which measures the fraction of light exiting from the fiber within a given solid angle. The value of FRD depends on the aperture angle (i.e. the focal aperture F/\#) by which the fiber is fed, and by the focal aperture accepted by the spectrometer. The VNIR fiber receives an F/4 input beam and feeds the spectrometer with an F/3.5 output beam. Therefore, the FRD losses are about $5 \%$ and total efficiency is about $85 \%$.

The light from the telescope can be fed to the fiber on the image plane or on the pupil plane. The former solution is used in HARPS, the ultra-high precision astronomical spectrometer which has reached the highest accuracy in the detection of extra-solar planets. On the other hand, pupil-feeding are often used in fiber-fed astronomical instruments. In the case of VNIR we can use both solutions, the only difference being the curvature of the input surface of the fiber, which is flat in case of image-feeding. For pupil-feeding, instead, the curvature is such that the first part of the fiber acts as a micro-lens adapter. We plan to test both solutions and select the one providing the best performances in terms of total efficiency and scrambling gain.

\section{The detector choice}

For the visible and near infrared channel, two options have been considered for VNIR in order to cover the (90 um) spectral resolution element on the focal plane: $512 \times 512$ matrix with $18 \mu \mathrm{m}$ square pixels (binning $5 \times 5$ ) and $256 \times 256$ format with $30 \mu \mathrm{m}$ square pixels (binning $3 \times 3$ ) Mercury Cadmium Telluride, MCT, operating at high frame rate (of the order of $10 \mathrm{~Hz}$ ). The first option is considered as baseline in this paper.

Table 3 Resuming data of encircled energy at focused and defocused system (WFE $250 \mathrm{rms}$ )

\begin{tabular}{lll}
\hline WFE rms @1 $\mu \mathrm{m}$ & $7.3 \mathrm{rms}$ & $250 \mathrm{rms}$ \\
Defocus shift M1-M2 (micron) & 0 & 87 \\
GEO radius spot (micron) & Airy radius 4.9 & 12.47 \\
$\begin{array}{l}\text { Encircled energy in-fibre diameter } \\
\quad(2 \text { arcsec) }\end{array}$ & $96.75 \%$ & $96.75 \%$ \\
\hline
\end{tabular}


MCTs have a good efficiency in the VNIR spectral range keeping a very low readout noise. Like other spectrometric EChO channels working in the infrared, the choice of an MCT permits the detector to work at a temperature around $40-45 \mathrm{~K}$, matching that of the optical bench of the modules. This fact will allow the instrument to have a very low thermal noise. From the performance point of view, readout noise, pixel size and dark current are the most crucial parameters that have been taken into consideration for the selection. This because the VNIR signal to noise ratio drops below $1 \mu \mathrm{m}$ and detector noise performances are crucial to meet the requirements. As far as the $512 \times$ 512 format is concerned, Selex and US manufacturers (Teledyne and Raytheon) offer comparable performances. While the US detectors appears to be in a mature state, Selex has a series of technical activities ongoing and planning to improve the performances of the VNIR detector, taking one of these devices at TRL 5 at beginning of 2015. From both the technical and programmatic information we have received from manufacturers, we assume Teledyne as baseline and Selex as a backup. Teledyne detectors can also be connected directly to the SIDECAR ASIC, chosen as baseline for the VNIR CFEE (as well as for the SWIR and FGS modules). This solution is better in terms of power consumption, thermal coupling and simplifies the overall harnessing between the detector and the CFEE and between CFEE and WFEE/ICU.

\section{Noise effects studies}

A study has been carried out to evaluate the best readout mode to adopt with the selected detector, taking into account the following main aspects: the need to minimise the equivalent noise in both bright and faint stars observations, the need to detect and correct for the cosmic rays hits effects and, finally, the need to simplify the on board data processing procedure in order to reduce the data rate and volume.

The MCT detectors allow for non-destructive readout modes, such that multiple readouts are possible without disturbing ongoing integration.

In Fig. 10 a non destructive readout sampling scheme is shown, for a single MCT pixel, in which the detectors integrating ramps are indicated in blu. In the sample upthe-ramp readout mode, the detectors readouts are equally spaced in time, sampling uniformly the ramp. By collecting all samples it is possible to fit the ramp slope. Provided that the number of samples is statistically significant, in case of cosmic rays hits, a jump or even a smooth modification of the slope can be detected and the corresponding samples rejected. This method is accurate but quite demanding in terms of real time processing power. In the multi-accumulate readout mode, only contiguous groups of samples are considered. The groups are equally spaced in time, but the samples between the groups are discarded. In Fig. 10 the samples groups are highlighted in red.

With reference to Fig. 2, where the expected location of the observed spectrum on the focal plane is reported, it can be seen that different pixels will be interested by different spectrum wavelengths and orders and, consequently, by different input flux levels. Considering the spectral types of the sources that will be observed by EChO, and convolving their flux in the various spectral channels with the channel bandpass and all other instrumental effects, included the detectors quantum efficiency, the obtained focal plane intensities in the range $0.6 \mu \mathrm{m}-2.5 \mu \mathrm{m}$ show a regular behaviour 


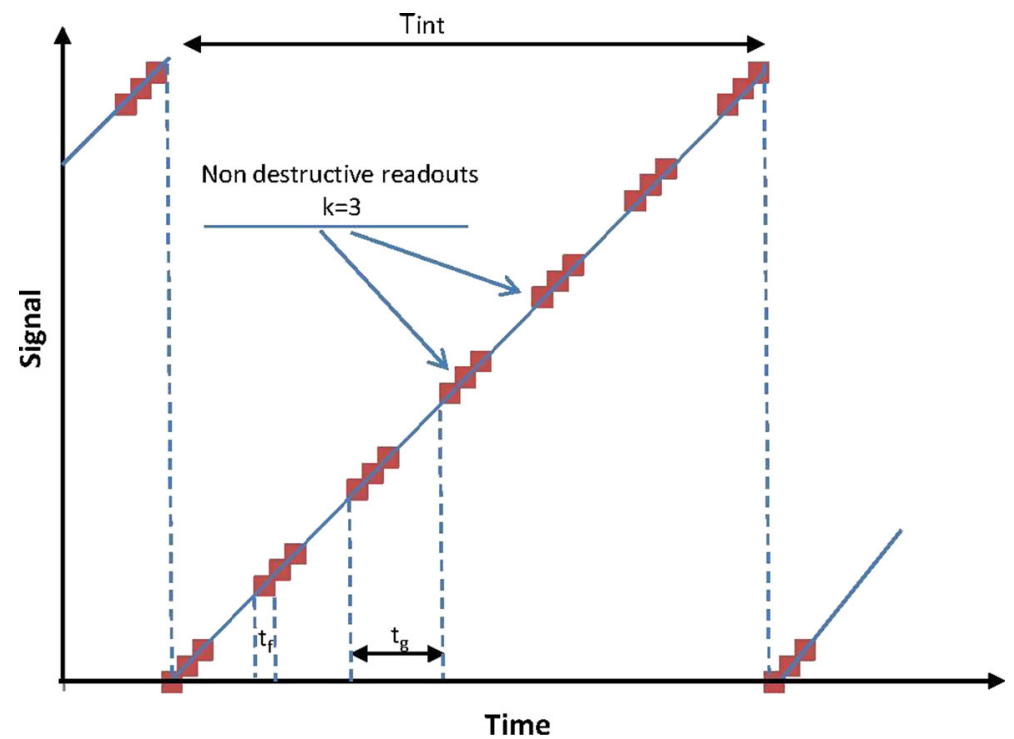

Fig. 10 Non destructive MCT detectors readout sampling scheme

with similar values in all considered spaxels (where spaxels are the equivalent of the spectral channels defined in section 3.1 in which pixels are binned in both the spectral and spatial directions) while in the range $0.4 \mu \mathrm{m}-0.6 \mu \mathrm{m}$ the expected flux is considerably lower. Given the early phase of the mission and the status of the design of the overall detectors data acquisition chain, in our present work we have used the same readout mode for all wavelengths. This assumption does not allow to optimise the results for the shorter wavelengths, but shall be considered as the first step of a more detailed investigation that will be performed in the next phases of the work.

The general expression for the total noise variance of an electronically shuttered instrument using the non-destructive readout can be computed using well known relations based on fundamental principles. It has been presented for the first time in its complete form by $[9,10]$ and is reported herein below:

$$
\sigma^{2}=\frac{12(n-1)}{k n(n+1)} R^{2}+\frac{6\left(n^{2}+1\right)}{5 n(n+1)}(n-1)\left(t_{g}\right) f-\frac{2\left(2 k^{2}-1\right)(n-1)}{k n(n+1)}(k-1)\left(t_{f}\right) f
$$

$R$ is the readout noise and $f$ is the flux, including photonic flux and dark currents. $R$ is in units of $e^{-} \mathrm{rms}$ and $f$ is in units of $e^{-} s^{-1}$ spaxe $^{1} ; k$ is the number of samples per group and $n$ is the number of groups per exposure. The frame time $t_{\mathrm{f}}$ is the time interval between the acquisition of two consecutive frames (frame sampling time). The group time $t_{g}$ is the time interval between the acquisition time of the first frame of one group and the first frame of the next group.

We used this relation to evaluate the noise expected for the VNIR detectors when read out using the sample up-the-ramp method. The result has then been compared with the system requirements for the two different detector arrays under study for the EChO mission, see [9]. The aim of the work has been to provide indications on how to optimize the EChO focal plane arrays sampling rate and data processing procedures in order to 
achieve the best signal to noise ratio and to identify and remove cosmic rays effects. The results of this activity will also be used to dimension the on-board data processing unit hardware and to define the architecture of the on-board data processing software.

The sampling rates of $8 \mathrm{~Hz}$ for bright sources and $1 / 16 \mathrm{~Hz}$ for faint sources have been considered to limit properly the overall data volume and processing resources (see [6]). The adopted integration times are $3 \mathrm{~s}$ for bright sources and $600 \mathrm{~s}$ for faint sources. Given the estimated input fluxes for the two types of sources, these times allowed to couple with the maximum detectors well capacity in both cases. All comparisons have been made assuming an operating temperature of $40 \mathrm{~K}$.

For bright sources, it was possible to obtain an optimized set of readout mode parameters only considering the Teledyne detectors this providing an expected total noise below the scientific requirement. The estimated noise for the Selex detectors was always well above the noise requirement and the obtained trend was not decreasing with the increase of the integration time.

In particular, in the case of the Teledyne detectors, for $k \geq 2$, the minimum $n$ to satisfy the requirement was always very low. This situation will allow to tune the overall measurement duration (max integration time) based only on the deglitching procedure performances, keeping it as short as possible, thus minimizing the expected number of cosmic hits.

In case of faint sources the results obtained for the $1 / 16 \mathrm{~Hz}$ sampling rate were similar to the previous ones, even if in this case the Selex detectors were able to meet the noise requirements in at least one case, with $k=3$ and the minimum $n$ equal to 7 .

Considering these preliminary results it was shown that with Teledyne sensors it is possible to better combine bright and faint sources results, while Selex detectors performances in terms of the overall noise obtainable with different readout strategies need to be better investigated. In particular, the main conclusion of our analysis is that two different readout rates and sampling methods are needed for bright sources and faint sources. With the noise performances considered for the Teledyne MCT detectors, it is possible to meet the noise requirements well within the maximum allowed integration times in both cases.

Future investigations are planned to improve the overall detector readout chain performances. The possibility to apply hardware coded ramps coaddition and to modify the detectors sampling rates will allow to explore a wider parameter space for the optimization of the readout mode procedures.

With respect to the cosmic hits effects, assumptions based on studies made for the JWST telescope (see $[11,12]$ ) give an expected rate of cosmic events with impact on the detector confined between 5 and 30 events $/ \mathrm{s} / \mathrm{cm}^{2}$. The estimated hit rates obtained for the whole VNIR focal plane array are reported in Table 4. Assuming that at least

Table 4 Expected cosmic rays hits rate for VNIR focal plane array positioned in Lagrangian point L2

\begin{tabular}{lll}
\hline Focal plane array (pixels) & $256 \times 256$ & $512 \times 512$ \\
Pixel size $(\mu \mathrm{m})$ & 30 & 18 \\
Mean rate $($ events/s) & 11 & 15 \\
Events in $600 \mathrm{~s}$ & 6600 & 9000 \\
Events in $3 \mathrm{~s}$ & 33 & 45 \\
Pixels affected in $3 \mathrm{~s}$ & $0.25 \%$ & $0.10 \%$ \\
Pixels affected in $600 \mathrm{~s}$ & $50.35 \%$ & $17.15 \%$ \\
\hline
\end{tabular}


five pixels per event are affected by each hit, the estimate of the expected percentage of data loss is reported in the last two rows of the Table.

It can be seen that, in case of bright sources, the percentage of pixels affected by the glitches will be very low and therefore it will be not necessary to correct the ramps for the cosmic hits effects, but it will be sufficient to identify and discard the affected readouts (only a max $0.25 \%$ of the overall array will be affected by the cosmic hits in a $3 \mathrm{~s}$ exposure). In case of faint sources a more detailed evaluation is needed, to confirm the necessity to implement a deglitching procedure onboard.

\section{The detector's electronics}

The MCT-based detector will be coupled with a ROIC (Read Out Integrated Circuit) bump bonded to the device's sensitive area. The ROIC will acts as a proximity electronics in order to extract the low level noise analogue signal from the detector, addressing the very low power dissipation requirements imposed by the environmental thermal aspects. The analogue signal will be amplified by the ROIC output OPAMP(s) (typically 4 or 8 for the two detector halves collecting respectively the VIS and NIR signals of the target spectrum) and fed to the cold front-end electronics (CFEE) where A/D conversion will take place.

The two detector halves OPAMPs gains shall be properly set in order to reach a proper signal level and maximize the $\mathrm{S} / \mathrm{N}$ ratio for the VIS and NIR spectra with respect to the acquired number of up-the-ramp collected samples, the detector's QEs and the overall instrumental efficiency in the VIS and NIR spectral bands. Another way to be explored and verified is connected to the possibility to set different ramp time lengths and samples number for the two detector halves, in order to reach the desired $\mathrm{S} / \mathrm{N}$ ratio. The latter solution could however complicate the clocks sequencing, the digital data acquisition timing and the overall detector management.

The payload's warm section electronics is essentially constituted by the warm frontend electronics (WFEE) generating driving signals for the detector ROIC/CFEE and the Instrument Control Unit (ICU) acting as the main payload processing electronics and collecting the digitized signals from all scientific channels. WFEEs will reside in a box specifically designed and located near the ICU which will be kept at a temperature in the range $0-40{ }^{\circ} \mathrm{C}$.

The detector is expected to be integrated easily and operate well with a range of electronics solutions. The distance between the Detector Sub Assembly and the CFEE and between the CFEE and the WFEE appears unavoidable in this system presentation and introduces technical challenges associated with a distributed signal chain including driving load capacitance, achieving settling, minimizing cross talk, ensuring stability and reducing noise.

The selected detector can be easily interfaced with the SIDECAR electronics solution, which helps to mitigate a number of electronics design challenges in implementing a full-functional solution. The key benefit is the closer integration of the ADCs to the detector which is expected to simplify the interface design, safeguard SNR and mitigate cross talk and some noise sources.

The baseline SIDECAR CFEE will receive a master clock and sync signals to be properly operated and to generate the detector clocks and control signals. The WFEE 
could also include an FPGA to provide a serial interface both to the SIDECAR and ICU and to perform pre-processing on scientific digital data (e.g. masking) and housekeepings.

The WFEE, if definitively adopted, shall implement stabilized voltage regulators and bias generators for the CFEE and the detector and shall interface the CFEE using a suitable cryo harness design $[6,13]$. This critical subsystem is designed as part of the signal interface between the detector, CFEE and WFEE in order to ensure that the best subsystem trades and required signals performance are achieved by design.

\section{Summary}

In the present paper the scientific objectives of the $\mathrm{EChO}$ mission have been presented. The VNIR module has been designed to fulfill both technical and scientific requirements of the proposed mission. Some of the adopted technical solutions have been shown.

Acknowledgments The authors wish to thank the Italian Space Agency for the financial support to the EChO programme. They are also grateful to the other members of EChO consortium and to the European Space Agency for their support during the phase A study. The present research has been funded by the contract ASI-INAF I/022/12/0.

Open Access This article is distributed under the terms of the Creative Commons Attribution License which permits any use, distribution, and reproduction in any medium, provided the original author(s) and the source are credited.

\section{References}

1. The Extrasolar Planet Encyclopaedia http://www.exoplanet.eu/ (2014). Accessed 9 Jan 2014

2. Tinetti, G., et al.: EChO. Exoplanet characterisation observatory. Exp. Astron. 34(2), 311-353 (2012)

3. Echo Team: Mission Requirements Document. http://sci.esa.int/echo/51293-echo-mission-requirementsdocument/ (2013). Accessed 7 Apr 2014

4. Capaccioni, F., et al.: VIS-NIR imaging spectroscopy of Mercury's surface: SIMBIO-SYS/VIHI experiment onboard the BepiColombo mission. In: IEEE Trans. Geosci. Remote Sens. 48, pp. 3932-3940 (2010)

5. Melchiorri, R., Piccioni, G., Mazzoni, A.: VIRTIS-M flight lamps. Rev. Sci. Instrum. 74, 3796-3801 (2003)

6. Focardi, M., Farina, M., Pancrazzi, M., Di Giorgio, A.M., Pezzuto, S., Ottensamer, R., Pace, E., Micela, G.: EChO electronics architecture and SW design. Experimental Astronomy EChO Special Issue (2014)

7. Lim, T.: EChO Instrument Calibration and Data Processing Plan. http://sci.esa.int/science-e/www/object/ doc.cfm?fobjectid=53425 (2013). Accessed 7 Apr 2014

8. Waldmann, I., Pascale, E.: EChO pointing jitter impact on photometric stability. http://sci.esa.int/sciencee/www/object/doc.cfm?fobjectid=53425 (2013). Accessed 3 Jan 2014

9. Farina, M., Di Giorgio, A.M., Focardi, M., Pace, E., Micela, G., Galli, E., Giusi, G., Liu, S.J., Pezzuto, S.: "Preliminary study of the EChO data sampling and processing". Proc. SPIE Astronomical telescopes and instrumentation, (in preparation) (2014)

10. Rauscher, B.J., Fox, O., Ferruit, P., et al.: Detectors for the James Webb Space Telescope near-infrared spectrograph I: readout mode, noise model, and calibration considerations. PASP 119, 768 (2007)

11. Fixsen, J., Offenberg, J.D., et al.: Cosmic-ray rejection and readout efficiency for large-area arrays. PASP 112, 1350-1359 (2000)

12. Rauscher, B.J., Isaacs, J.C.: "Cosmic ray management on NGST 1: the effect of Cosmic Rays on Near Infrared Imaging Exposure Time", STScI-NGST-R-0003A (2000)

13. Morgante, G., Terenzi, L.: TMM/GMM description and results. http://sci.esa.int/science-e/www/object/ doc.cfm?fobjectid=53425 (2013). Accessed 7 Jan 2014 